\title{
Governança Colaborativa em Instituições de Ensino: Uma Quasi- Revisão Sistemática da Literatura
}

\author{
Danilo Ferreira Neves - PROCC/UFS - danilo.neves@ufs.br \\ Lidiany Cerqueira Santos - UFS - lidianycs@ufs.br \\ Mylena Alves Santos - UFS - myle_alves@hotmail.com \\ Methanias Colaço Rodrigues Júnior - PROCC/UFS - mjrse@ hotmail.com
}

Resumo: Contexto. Tecnologias para estimular a participação eletrônica vem sendo adotadas por governos como forma de legitimar direitos, promover transparência e resolver problemas de forma mais eficiente. Embora validada no cenário político, a governança colaborativa ainda é pouco adotada em centros acadêmicos. Objetivo. Entender e mapear como práticas colaborativas estão sendo adotadas na governança de instituições de ensino. Metodologia. Foi elaborada uma Quasi-revisão sistemática da literatura. Resultado. A busca selecionou 3 estudos entre 526. Após análise, foram identificados nos estudos os recursos pertinentes para viabilização de colaboração, as práticas de participação utilizadas e como a inserção desse modelo administrativo influenciou na qualidade de ensino e no desenvolvimento da comunidade acadêmica. Conclusão. Pelo caráter metodológico aplicado, o trabalho serve como um guia, identificando tendências e sintetizando práticas que podem ser replicadas em novos projetos.

Palavras-chave: Participação Eletrônica, Democracia, Comunidade Acadêmica, Governança Colaborativa.

\section{Collaborative Governance in Educational Institutions: A Quasi-Systematic Literature Review}

Abstract: Context. Technologies to enable electronic participation are being adopted by governments as a way to legitimize rights, promote transparency and solve problems more efficiently. Although validated in the political arena, collaborative governance is still poorly adopted in academic centers. Objective. Understand and map how collaborative practices are being adopted in the governance of educational institutions. Methodology. A systematic quasi-literature review was developed. Results. The search selected 3 studies from 526. Through analysis, the studies identified the relevant resources to enable collaboration, the participation practices used and how the insertion of this administrative model influenced the quality of teaching and the development of the academic community. Conclusion. Based on the methodological, the work serves as a guide, identifying trends and synthesizing practices that can be replicated in new projects.

Keywords: Electronic Participation, Democracy, Academic Community, Collaborative Governance.

\section{Introdução}

O avanço da Tecnologia da Informação e Comunicação (TIC) nas instituições públicas permitiu ao governo reduzir seus níveis hierárquicos e estabelecer novas políticas de relacionamento Estado-Sociedade. As novas tecnologias se tornaram um verdadeiro catalisador de mudanças nas estruturas dessas instituições. Suas iniciativas são consideradas uma promoção da acessibilidade, construindo uma cultura de transparência, engajamento civil e combate à corrupção (Janowski, 2015; West, 2004). DOI: 
O surgimento desses novos canais de comunicação sinalizou o desenvolvimento de novos modelos de interação, estreitando os lanços entre a população e seus gestores. Nesse sentido, o cidadão passa a ser notado não somente como um usuário, mas também como um colaborador, tendo suas ideias e opiniões integradas ao conselho de gestão, elevando a qualidade dos serviços públicos e trazendo maior controle social (Freitas e Dacorso, 2014).

Aos poucos, o cidadão é incluído no planejamento público passando a atuar em diferentes áreas da administração: planejamento urbano, fiscalização orçamentária, elaboração e revisão de políticas públicas (Neves et al., 2019). Os benefícios gerados por essa união são classificados por Hilgers e Piller (2011) quando citam o aumento da aceitação das ações do governo, também por Bertot et al. (2010) que indicam o aumento da responsabilidade fiscal e também no debate de West (2004) que crê na formação de uma população mais interessada em entender e participar dos processos políticos.

Embora a ideia de participação colaborativa esteja bem representada em outros setores, ela ainda se mostra tímida na educação. A inclusão de estudantes em processos de tomada de decisão em universidades e escolas é tida como limitada. E normalmente, quando existe essa interação, os estudantes são representados por centros estudantis, uma prática insuficiente avaliada sobre um espectro democrático (Lizzio e Wilson, 2009).

Compreendendo os benefícios que a participação eletrônica e a democracia digital proporcionaram no setor público e político, esse estudo tem como objetivo mapear, por meio de uma Quasi-Revisão Sistemática da Literatura (QRSL), as ferramentas de participação eletrônicas nas instituições de ensino, e quais estratégias estão sendo adotadas na implantação desses sistemas. Desta maneira, as ferramentas foram classificadas quanto aos seus recursos, o nível de interação, as práticas utilizadas para engajamento e colaboração e, por fim, quais os benefícios gerados para educação. Esse artigo foi estruturado da seguinte forma: a Seção 2 detalha a metodologia utilizada; a Seção 3 traz a análise dos resultados e a Seção 4 exibe a conclusão e as considerações finais.

\section{Metodologia}

Uma QRSL é um estudo secundário, baseado em evidências, que tem como proposta o levantamento, a identificação, avaliação e classificação da literatura para responder questões de pesquisa específicas (Kitchenham, 2004). Diante disso, o protocolo utilizado nesse trabalho segue as diretrizes de revisão sintetizadas por Kitchenham (2004). Embora a QRSL cumpra todas as fases de avaliação da revisão sistemática, ela não é considerada como tal pela falta de uma linha base para meta-análise, lhe faltando em seu estudo modelos de comparação para os resultados (Travassos et. al., 2008). A QRSL deste trabalho foi desenvolvida em três fases: Planejamento, Condução e Relatório Final da Revisão.

- Fase de Planejamento: Neste primeiro momento são estabelecidos as necessidades e o direcionamento da pesquisa. Sendo assim, são definidos o objetivo, as questões de pesquisa, a validação do escopo de pesquisa e um protocolo de busca e seleção de estudos. Este protocolo deve especificar onde serão buscados os artigos, a metodologia utilizada e os critérios para inclusão ou exclusão dos estudos encontrados.

- Fase de Condução: Essa etapa se inicia com a execução dos termos definidos na fase de planejamento. Os artigos identificados são analisados, avaliados e sintetizados com base no escopo de pesquisa e os critérios estabelecidos. 
- Fase de Relatório da Revisão: Nessa última fase, é feita uma amostra dos dados obtidos nas fases anteriores e expostas as ações tomadas para mitigar as ameaças à validade da revisão.

O processo foi construído em par com auxílio de uma pesquisadora mestre da área de educação e informática. A Figura 1 apresenta o processo de revisão e as atividades executadas em cada uma das fases.

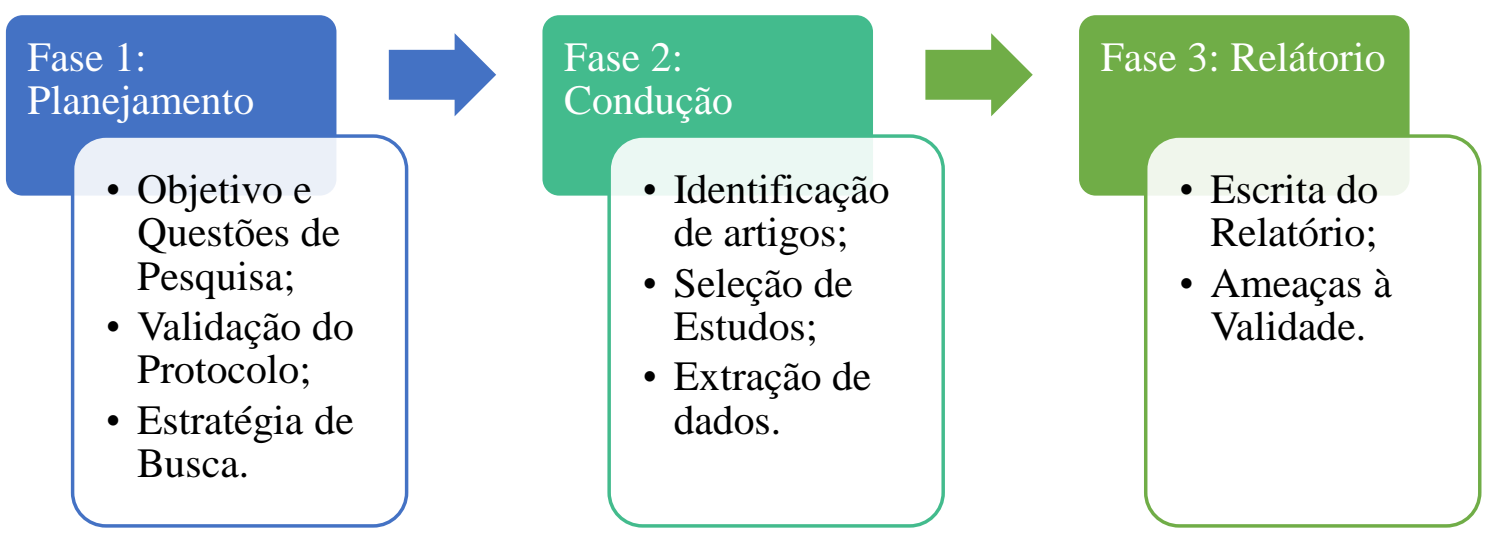

Figura 1 - Processo de revisão sistemático (Brereton et al., 2007).

\subsection{Fase 1: Planejamento}

O planejamento iniciou com a definição do objetivo e das questões de pesquisa. Em seguida, uma estratégia de busca foi construída, determinando as bases, os termos de busca e os critérios de inclusão e exclusão dos artigos.

\subsubsection{Objetivo e Questões de Pesquisa}

Esse estudo tem como objetivo levantar e avaliar os modelos de participação eletrônica aplicados dentro de instituições educacionais de ensino e, deste modo, expor as estratégias que compõem uma governança colaborativa para estudantes, professores e administradores. Para alcançar esse objetivo foram definidas as seguintes questões de pesquisa:

- QP1: Quais os recursos utilizados para viabilizar a participação colaborativa entre gestão e comunidade acadêmica?

- QP2: Quais as práticas utilizadas para empoderamento de estudantes perante a governança das instituições de ensino?

- QP3: Quais os resultados para o ensino e aprendizagem que as técnicas proporcionam?

\subsubsection{Validação do Escopo de Pesquisa}

Uma busca prévia foi realizada para averiguar se o posicionamento do estudo compete ao campo de pesquisa atual, avaliar às QP e ter subsídios para classificação das ferramentas. Foram buscados trabalhos que trazem uma análise geral da área e fornecem mecanismos para caracterização de ferramentas de participação. Foram utilizados os seguintes estudos:

- Towards an evaluation framework for eParticipation (Macintosh e White, 2008).

- The shape of eParticipation: Characterizing an emerging research area (Sæbø et al., 2008).

\subsubsection{Fontes de Dados}


Para levantamento dos estudos foram utilizadas as seguintes bases de dados: IEEE Xplore, Compendex, Scopus, Web Science, Science Direct e ACM Digital Library. Essas bases foram escolhidas por serem importantes indexadores de publicações cientificas, principalmente da área de computação. Ademais, seus motores de busca automatizados possuem maior eficiência em encontrar estudos relevantes (Kitchenham et al., 2009).

\subsubsection{Termos de Busca}

Para compreender as terminologias da área de pesquisa foram usados os estudos de validação descritos na Seção 2.1.2. As palavras-chaves foram agrupadas de acordo com o modelo sugerido por Kitchenham (2004): População, Intervenção, Comparação e Outcome (PICO). A Tabela 1 apresenta os termos identificados e distribuídos de acordo com o modelo.

Tabela 1 - Termos de busca.

\begin{tabular}{lll}
\hline Categorias & Descrição & \\
\hline População & $\begin{array}{l}\text { Student, School, College, University, Education } \\
\text { Intervenção }\end{array}$ & $\begin{array}{l}\text { Tool, Tools, Technologies, Technology, Application, } \\
\text { Plataform, Approach, Model, Framework. }\end{array}$ \\
Comparação & -- & \\
Outcome (Resultado) & $\begin{array}{l}\text { e-Participation, e-Democracy, eletronic participation, } \\
\text { eletronic participative, eletronic democracy. }\end{array}$ \\
\hline
\end{tabular}

Com base nos termos de busca foi elaborado a seguinte string genérica de busca:

STRING DE BUSCA: (school OR college OR university OR student OR education) AND (e-participation OR e-democracy) AND (tool* OR technolog* OR application OR plataform OR approach OR model OR framework)

\subsubsection{Critérios de Seleção}

Os critérios de inclusão e exclusão são utilizados para delimitar estudos mais relevantes para às questões de pesquisa. Foram definidos os seguintes critérios de inclusão:

[IC1]: O estudo deve conter os termos de busca em seu título, resumo ou palavraschaves.

[IC2]: O estudo deve explorar uma ferramenta, com valores de participação eletrônica, utilizada para integrar estudantes em um modelo de gestão participativo.

[IC3]: O estudo deve estar disponível para pesquisa online.

Os critérios de exclusão:

[EC1]: Estudos publicados antes de 2009.

[EC2]: Estudos sem relação com participação eletrônica ou democracia eletrônica. [EC3]: Estudos secundários.

[EC4]: Estudos preliminares.

[EC5]: Estudos duplicados.

\subsection{Fase 2: Condução da Revisão}


A busca foi executada no segundo semestre de 2019. A quantidade de artigos identificados, o método de filtragem e a extração dos dados são detalhados a seguir.

\subsubsection{Estudos Encontrados}

A string de busca foi adaptada e executada em cada um dos indexadores. Os motores de busca foram configurados para publicações a partir do ano de 2009 e que contivessem no título, resumo ou palavras chaves algum dos termos de busca. Foram encontrados um total de 526 artigos. A relação de artigos encontrados por veículos é apresentada na Tabela 2.

Tabela 2 - Relação de veículos e publicações identificados.

\begin{tabular}{c|c}
\hline Veículos & Estudos \\
\hline ACM & 111 \\
Compendex & 109 \\
IEEE & 79 \\
ScienceDirect & 55 \\
Scopus & 97 \\
Web Science & 75 \\
\hline Total & 526 \\
\hline
\end{tabular}

\subsubsection{Seleção de Estudos}

A seleção de estudos foi realizada em duas etapas. Na primeira, foi feito a leitura do título e resumo dos estudos, já na segunda etapa, os estudos foram lidos por completo.

$\mathrm{Na}$ primeira fase da seleção, os estudos foram avaliados pelo resumo. A seleção se atentou ao objetivo do trabalho e sua conclusão, identificando o alinhamento do artigo ao escopo da quasi-revisão. Após a realização da leitura, dentre os 526 artigos, 21 foram selecionados para leitura completa.

$\mathrm{Na}$ segunda fase, os 21 artigos selecionados na etapa de leitura de resumo foram analisados por completo. A leitura dos artigos avaliou a capacidade dos estudos em responder às questões de pesquisa. Nessa etapa, um maior rigor foi adotado e apenas trabalhos que detalhassem as funções e os resultados do uso das ferramentas foram considerados. Dentre os 21 estudos selecionados, apenas 3 artigos atenderam aos requisitos da pesquisa. $\mathrm{Na}$ Tabela 3 , é apresentada a distribuição dos estudos por repositório científico e a quantidade de artigos selecionados em cada fase.

\begin{tabular}{c|c|c|c}
\multicolumn{4}{|c}{ Tabela 3-Artigo selecionados por bases. } \\
\hline Veículos & $\begin{array}{c}\text { Estudos } \\
\text { Encontrados }\end{array}$ & $\begin{array}{c}\text { Após Leitura do } \\
\text { Resumo }\end{array}$ & $\begin{array}{c}\text { Após Leitura } \\
\text { Completa }\end{array}$ \\
\hline ACM & 111 & 0 & 0 \\
Compendex & 109 & 5 & 1 \\
IEEE & 79 & 3 & 0 \\
ScienceDirect & 55 & 0 & 0 \\
Scopus & 97 & 2 & 2 \\
Web Science & 75 & 11 & 0 \\
\hline Total & 526 & 21 & 3 \\
\hline
\end{tabular}

\subsection{Fase 3: Relatório da Revisão}

A cada fase de filtragem uma quantidade significante de artigos foi excluída. Foram eliminados artigos duplicados, que não atendiam aos critérios de inclusão, com baixa aderência ao escopo da pesquisa e, por fim, artigos que não eram capazes de trazer respostas às questões de pesquisa. No final do processo de seleção, restaram apenas 3 
artigos, correspondendo a 1,7\% do total inicial de estudos. Uma breve descrição de cada ferramenta é apresentada a seguir e sintetizada na Tabela 4:

- O MyUniversity é uma solução web resultado de uma iniciativa da União Europeia para instituições de ensino superior. A ferramenta integra todas as universidades parceiras em um superportal, criando um espaço de diálogo entre as diferentes comunidades, de países diferentes. Cada universidade ainda conta com um ambiente local próprio, para discutir diretrizes internas.

- "Universidade Conectada" é uma aplicação móvel desenvolvida pelo Programa de Pós-Graduação em Informática Aplicada da Universidade Federal Rural de Pernambuco. $\mathrm{O}$ aplicativo funciona como uma plataforma de manifestação, através do celular os estudantes podem reportar problemas de infraestrutura, ensino e pesquisa para os gestores. A manifestação acompanha a localização geográfica e possibilita que fotos do problema sejam anexadas.

- O Liberopinion é uma plataforma web desenhada para unir a comunidade acadêmica portuguesa em um espaço deliberativo de discussão. A plataforma funciona como um mediador, concentrando ideias e direcionando ações e ainda conta com um portal próprio, o Liberopinion fornece ferramentas que podem ser integradas a sistemas de terceiros.

Tabela 4 - Ferramentas de participação eletrônica catalogadas.

\begin{tabular}{|c|c|c|c|c|}
\hline Ferramentas & $\begin{array}{c}\text { Tipo de } \\
\text { Instituição }\end{array}$ & País/Região & Atores & Referência \\
\hline MyUniversity & $\begin{array}{l}\text { Ensino } \\
\text { Superior }\end{array}$ & $\begin{array}{c}\text { Países da União } \\
\text { Europeia }\end{array}$ & $\begin{array}{c}\text { Estudantes, } \\
\text { Professores, } \\
\text { Gestores. }\end{array}$ & $\begin{array}{c}\text { (Cucurull, } \\
\text { Álvaro e } \\
\text { Puiggalí, } \\
\text { 2013) }\end{array}$ \\
\hline $\begin{array}{l}\text { Universidade } \\
\text { Conectada }\end{array}$ & $\begin{array}{l}\text { Ensino } \\
\text { Superior }\end{array}$ & Brasil & Estudantes. & $\begin{array}{c}\text { (Teixeira et al. } \\
\text { 2017) }\end{array}$ \\
\hline Liberopinion & Todas & Portugal & $\begin{array}{c}\text { Estudantes, } \\
\text { Professores, } \\
\text { Sindicatos, } \\
\text { Gestores. }\end{array}$ & $\begin{array}{c}\text { (Sousa et al., } \\
\text { 2014) }\end{array}$ \\
\hline
\end{tabular}

Para análise de crescimento da área de pesquisa, a Figura 2 mostra a quantidade de artigos encontrados em cada ano. É possível notar um crescimento acentuado a partir do ano de 2014, o alto índice de publicações foi mantido nos anos seguintes tendo 2016 como o ano com mais publicações. Embora ocorra uma queda brusca em 2019, é importante atentar-se ao fato de que este ano foi representado por publicações ocorridas apenas em seu primeiro semestre e não refletem no estado de relevância, alcançado gradualmente durante os anos, do campo de pesquisa.

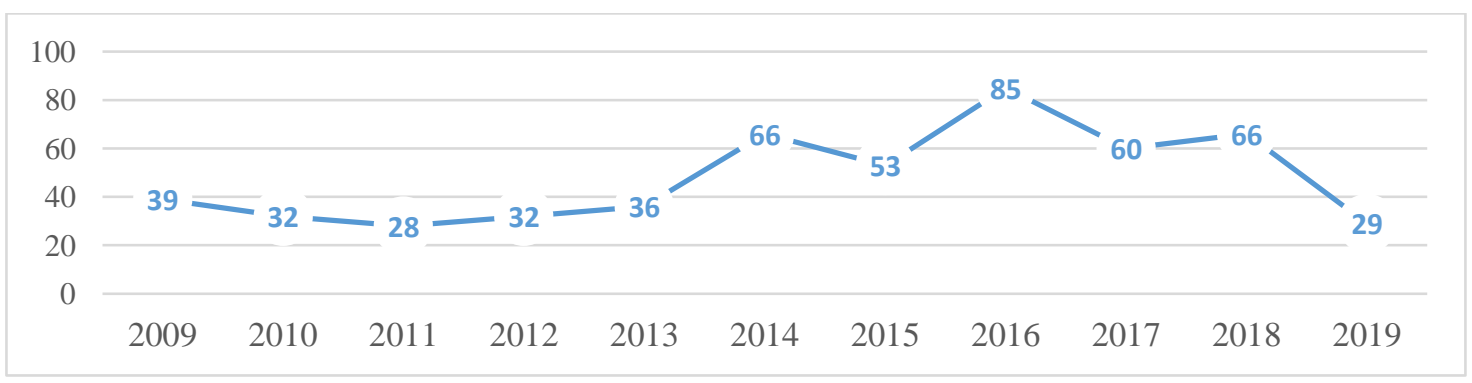

V. $17 \mathrm{~N}^{\mathrm{o}}$ 3, dezembro, 2019

Figura 2 - Estudos encontrados por ano.

DOI: 


\subsection{Ameaças à Validade}

\subsubsection{Validade de Construção}

A string de busca e a questões de pesquisa podem não cobrir estudos relevantes na área. Para mitigar essa ameaça, as palavras-chaves e as questões de pesquisa foram elaboradas a partir de uma pesquisa prévia no assunto e de artigos que estabelecem o estado da arte da linha de pesquisa (Seção 2.1.2).

\subsubsection{Validade Interna}

A quasi-revisão pode apresentar um resultado impreciso, trazendo estudos diferentes caso fosse executada novamente, dessa forma, para resolver esse problema, foi adotada uma metodologia para revisão sistemática definida por Kitchenham (2004). Conflitos na seleção de artigos foram discutidos entre os pesquisadores para mitigar o viés pessoal na seleção dos estudos.

\subsubsection{Validade Externa}

A busca pode não conter artigos relevantes para o estudo. Como forma de mitigar essa ameaça, a busca foi replicada em 6 bibliotecas digitais.

\section{Resultados}

Para melhor entendimento e visualização dos resultados, essa seção foi seccionada de acordo com às questões de pesquisa.

\subsection{QP1 - Quais os recursos utilizados para viabilizar a participação colaborativa entre gestão e comunidade acadêmica?}

Para responder essa QP consideramos o estudo de caracterização de ferramentas de participação formatado por Sæbø et al.(2008). Os recursos que viabilizam a operação de participação são descritos pelo autor como Fatores de Contexto. Tais fatores tanto podem ser tecnologias, quanto podem ser ações afirmativas da organização em favor da ferramenta. Para Sæbø et al.(2008) esses recursos são: Acessibilidade, Disponibilidade de Informação, Tecnologias Integradas a Solução, Plataforma, Questões Políticas e Influencia da Organização. Na Tabela 5 são apresentados os recursos encontrados em cada ferramenta.

Tabela 5 - Recursos e arquitetura utilizados para viabilizar a ação da ferramenta.

\begin{tabular}{|c|c|c|}
\hline Ferramentas & Plataforma & Recursos Destacados \\
\hline MyUniversity & Web & $\begin{array}{l}\text { Acessibilidade: } \\
\text { • Integração com Redes Sociais. } \\
\text { Disponibilização de Informação } \\
\text { • Notícias; } \\
\text { - Calendários de evento. }\end{array}$ \\
\hline $\begin{array}{l}\text { Universidade } \\
\text { Conectada }\end{array}$ & Aplicação Móvel & $\begin{array}{l}\text { Acessibilidade: } \\
\text { - O aplicativo opera offline, } \\
\text { guardando os dados para serem } \\
\text { enviados quando conectado à } \\
\text { internet. } \\
\text { Tecnologias Integradas a Solução: } \\
\text { - Geolocalização; } \\
\text { - Integração com a câmera do } \\
\text { celular. }\end{array}$ \\
\hline Liberopinion & $W e b$ & Acessibilidade: \\
\hline
\end{tabular}




\begin{tabular}{l|l|l}
\hline & $\begin{array}{l}\text { Disponibiliza interface para } \\
\text { integração com outros portais. }\end{array}$ \\
& $\begin{array}{c}\text { Disponibilização de Informação: } \\
\bullet \\
\end{array}$ \\
& Notícias; \\
\hline
\end{tabular}

Analisando os recursos identificados, é evidente a preocupação com o acesso as plataformas. No contexto de ferramentas de participação, a acessibilidade diz respeito a quantas pessoas poderão acessar a plataforma e de quais maneiras será acessada (Macintosh, 2004). A coleta é fundamentada, tendo em vista que o recurso é considerado uma forma de democratização da informação e integração de grupos heterogêneos, a precarização do recurso pode servir de gatilho para excluir grupos e abrir espaço para fraudes, quando se privilegia o acesso ou o restringe totalmente (Sæbø et al., 2008).

\subsection{QP2 - Quais as práticas utilizadas para empoderamento de estudantes perante a governança das instituições de ensino?}

No modelo de Sæbø et al.(2008), uma ferramenta com propósitos democráticos pode fornecer a seus usuários opções como: Votação Eletrônica, Espaço Virtual de Debate, Tomada de Decisão Unificada, Ativismo eletrônico, Manifestação de Irregularidades, Criação de Eventos e Criação de Petições Eletrônicas.

Ao todo, as ferramentas apresentaram 4 serviços de participação eletrônica. $\mathrm{O}$ MyUniversity e a Liberopinion se mostraram as plataformas mais completas, suas funções indicam a presença de 3 atividades ligadas a participação eletrônica. A Universidade Conectada foi avaliada com apenas uma atividade. A relação de práticas é apresentada na Tabela 6.

Tabela 6 - Funcionalidades relacionadas à Participação Eletrônica.

\begin{tabular}{c|c}
\hline Práticas de Participação Eletrônica & Ferramenta \\
\hline Espaço de Debate & MyUniversity, Liberopinion \\
\hline Manifestação de Irregularidade & $\begin{array}{c}\text { MyUniversity, Universidade } \\
\text { Conectada }\end{array}$ \\
\hline Petição & MyUniversity, Liberopinion \\
\hline Votação & Liberopinion \\
\hline
\end{tabular}

As práticas de participação de cada ferramenta demonstram o nível de interação que pretendem estabelecer com seus usuários. Macintosh (2004) dimensiona a participação eletrônica em 3 níveis: Informação, quando a instituição libera dados e informa a população de suas ações; Consulta, ocorre quando o mecanismo de interação permite a coleta da opinião do ponto de vista dos usuários; e Participação Ativa, na qual o usuário é totalmente integrado em um ambiente de discussão, fazendo parte diretamente do planejamento.

O Liberopinion e o MyUniversity são exemplos de ferramentas de Participação Ativa. O espaço de debate disponibilizado por elas permite que o usuário faça parte integralmente da governança da instituição, que no caso da Universidade Conectada, a ferramenta não emprega uma comunicação tão próxima com a comunidade. Disponibilizando um modelo de manifestação de problemas, a aplicação é dimensionada em um nível de Consulta. 


\subsection{QP3 - Quais os resultados para o ensino e aprendizagem as técnicas proporcionam?}

Os estudos indicam a aproximação dos membros da instituição, reduzindo tempo e custos de comunicação, além de um aumento no interesse dos participantes em contribuir com a administração.

Ao permitir que estudantes participem do processo de decisão, um sentimento de pertencimento é construído (Lizzio e Wilson, 2009). Essa autonomia, em um ambiente que suas retificações serão abalizadas, é um percussor para construção de uma consciência política e cidadã, preparando esses indivíduos para uma sociedade pluralizada (Kouba, 2017).

Além disso, sistemas como a "Universidade Conectada" atuam diretamente na promoção da qualidade da educação. Ou seja, por meio do aplicativo o estudante é capaz de alertar a gestão para a falta de livros na biblioteca ou um laboratório sucateado.

\section{Conclusão}

Esse trabalho teve como objetivo o mapeamento e a discussão de mecanismos utilizados para construção de uma governança colaborativa em instituições de ensino. A QRSL foi adotada como método de pesquisa, o modelo foi desenvolvido a partir do protocolo definido por Kitchenham (2004). A busca, realizada em 6 bibliotecas digitais, teve como resultado 526 artigos, refinados através de duas fases de leituras, nas quais foram aplicados os critérios de exclusão e inclusão. Na primeira fase, foram selecionados 21 estudos. Na segunda fase, após leitura completa, apenas 3 artigos foram selecionados para extração de dados e discussão.

O levantamento identificou um grande uso de recursos direcionado a acessibilidade, tendo sido constatado em todas ferramentas (QP1). Dentre as 3 ferramentas, 2 foram categorizadas como sistemas de Participação Ativa, onde os participantes interagem diretamente com os gestores, indicando um desejo contundente de integrar a comunidade (QP2). O nível de participação e abertura proporcionados por essas ferramentas, constatados na questão anterior, são os motores para construção dos resultados obtidos na QP3, onde os trabalhos relataram o aumento do engajamento entre os participantes e o estreitamento da comunicação, benefícios alcançados pela presença de espaços democráticos.

Visualizamos, com base nos resultados dessa revisão, a oportunidade de desenvolvimento de governanças colaborativas no ambiente escolar. Os dados coletados indicam a conformidade dos projetos com os sistemas desenvolvidos em outros campos, e seus benefícios em relação à comunicação, fiscalização e transparência, além de ser um catalizador de transformações interpessoais e organizacionais.

\section{Referências}

BERTOT, J. C., JAEGER, P. T., GRIMES, J. M. Using ICTs to create a culture of transparency: E-government and social media as openness and anti-corruption tools for societies. Government Information Quarterly. 2010.

BRERETON, PEARL, et al. "Lessons from applying the systematic literature review process within the software engineering domain." Journal of systems and software 80.4. 2007.

CUCURUll, J., Álvaro, A., PUIGGAlÍ, J. MyUniversity: E-participation and decision making for higher education. Electronic Government and Electronic Participation-Joint Proceedings of Ongoing Research of IFIP EGOV and IFIP ePart 2036. 2013. 
FREITAS, R. K. V. DE, DACORSO, A. L. R. Inovação aberta na gestão pública: análise do plano de ação brasileiro para a Open Government Partnership. Revista de Administração Pública 48(4). 2014.

HILGERS, D., \& PILLER, F. A government 2.0: fostering public sector rethinking by open innovation. Innovation Management, 1(2), 1-8. 2011

JANOWSKI, T. Digital government evolution: From transformation to contextualization. Government Information Quarterly, 32(3), 221-236. 2015.

KOUBA, K. "Determinants of student participation in higher education governance: the case of student turnout in academic senate elections in Czechia." Higher Education 76.1. 2018.

KITCHENHAM, B. Procedures for performing systematic reviews. Keele, UK, Keele University, 33(2004), 1-26. 2004

KITCHENHAM, B, et al. "The impact of limited search procedures for systematic literature reviews - A participant-observer case study,". 3rd International Symposium on Empirical Software Engineering and Measurement, Lake Buena Vista, FL. 2009.

LIZZIO, A., KEITHIA W. "Student participation in university governance: the role conceptions and sense of efficacy of student representatives on departmental committees." Studies in Higher Education 34.1. 2009.

MACINTOSH, A. Characterizing e-participation in policy-making. In 37th Annual Hawaii International Conference on System Sciences. IEEE. 2004.

MACINTOSH, A. AND WHYTE, A. "Towards an evaluation framework for eParticipation". Transforming Government: People, Process and Policy, Vol. 2 No. 1, pp. 16-30. 2008.

NEVES, D. F., SANTOS, L. C., NETO, A. J. A., JUNIOR, M. C. R. Social Control through Collaborative Tools: A Systematic Literature Mapping. In 16th CONTECSIInternational Conference on Information Systems and Technology Management. 2019.

SÆBØ, Ø., ROSE, J., FLAK, L. S. The shape of eParticipation: Characterizing an emerging research area. Government Information Quarterly, 25(3), 400-428. 2008.

SOUSA, A. A., et al. "Model of Digital Mediation to Support Communication between Teachers Unions and the Education Community." International Conference on Electronic Government and the Information Systems Perspective. Springer, Cham, 2014.

TEIXEIRA, M. M., et al.. Mobility, Cyberculture, App and Digital Citizenship: A Case Study of "Universidade Conectada". In World Conference on Information Systems and Technologies (pp. 503-510). Springer, Cham. 2017.

TRAVASSOS, G. H., et al. "An environment to support large scale experimentation in software engineering." 13th IEEE International Conference on Engineering of Complex Computer Systems (ICECCS 2008). IEEE, 2008.

WEST, D. M. E-government and the transformation of service delivery and citizen attitudes. Wiley Online Library. 2004. 